Respiration 2017;93:228-229

DOI: $10.1159 / 000455880$

\section{Investigating the Etiology of Bronchiectasis: You Do Not Find What You Do Not Look For}

\author{
Francesco Amati $^{\mathrm{a}}$ Elisa Franceschi ${ }^{\mathrm{a}}$ Andrea Gramegna ${ }^{\mathrm{a}}$ \\ James D. Chalmers ${ }^{b}$ Stefano Aliberti ${ }^{a}$ \\ ${ }^{a}$ Department of Pathophysiology and Transplantation, \\ University of Milan, Cardio-Thoracic Unit and Adult Cystic \\ Fibrosis Center, Fondazione IRCCS Cà Granda Ospedale

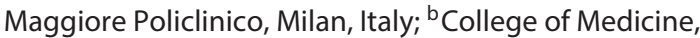 \\ University of Dundee, Dundee, UK
}

We read with great interest the manuscript by Buscot and coworkers [1], who retrospectively analyzed the etiology of bronchiectasis in an adult population of French subjects. We congratulate the authors for their effort to collect for the first time in the literature these data from France, which nicely add to those recently published from 7 other European countries [2]. As we clearly recognize the importance of identifying the etiology of bronchiectasis and especially those conditions that might change patients' management, we also share with Buscot and colleagues the concerns on the current approach in both testing bronchiectasis patients and presenting scientific data on this topic.

First, the absence of an animal model and a lack of basic science research in bronchiectasis limits our understanding of pathophysiology and leaves us groping in the dark using terms such as "cause of," "association with," "overlap between," or "correlation." Second, it is unknown if some of the currently reported etiologies of bronchiectasis, such as non-tuberculous mycobacteria or allergic bronchopulmonary aspergillosis, succeed versus precede bronchiectasis development. Both are likely. Third, most of the available literature does not evaluate the possibility that more than one predisposing factor might exist in the same patient, and the interaction between different conditions causing bronchiectasis is unknown. Fourth, although post-infective bronchiectasis accounts for the majority of "known" causes of bronchiectasis in most of the published cohorts, this definition suffers from several biases. There is consensus on neither the type and severity of previous infective processes which justify the development of bronchiectasis nor the acceptable time between them which should fit the "post-infective" definition. A patient having left lower lobe pneumonia who subsequently presents with left lower bronchiectasis 1 year later might receive the diagnosis of post-infective bronchiectasis, but do we know that the pneumonia did not occur due to an existing bronchiectasis in this lobe? More commonly, patients give a remote history of infection such as childhood pneumonia or whooping cough and are told this is the cause of their bronchiectasis, even when this event was 40 or 50 years earlier than the onset of their symptoms. In light of the median age of bronchiectasis patients (around 68 years according to unpublished EMBARC data), a clear recall bias might exist when a physician is investigating lower respiratory tract infections which occurred during patient's infancy or adolescence [3]. Furthermore, a reasonable geographical variability characterizes this etiology, mainly accounting for post-TB cases, as documented by a broad range from $62.5 \%$ in Africa to $31.2 \%$ in Europe and $18.9 \%$ in North America [4]. Fifth, the association between COPD and bronchiectasis has not been fully investigated and the different ways to diagnose these two conditions (functional vs. radiological diagnosis) make things much more complicated. Thus, different patients are currently classified in clinical practice under the same umbrella of bronchiectasis-COPD overlap syndrome: subjects with bronchiectasis and airflow obstruction (even if they are non-smokers), those with a prior diagnosis of COPD who develop bronchiectasis, and sometimes even patients without airflow obstruction, but having bronchiectasis and a smoking history [5]. Last but not least, data on the prevalence of bronchiectasis etiology published so far are limited not only by the retrospective nature of the study designs but also by the absence of the denominator used to calculate those rates (the number of tests performed). This represents a key issue that might alter the perception of the causes of bronchiectasis and explains why the wide range of idiopathic bronchiectasis detected across centers is, in reality, strictly dependent on the intensity of the workup performed. You do not find what you do not look for.

In light of these and other concerns as well as the absence of evidence on this subject, there is no consensus on what would be the minimum bundle of tests to be performed once the radiological diagnosis of bronchiectasis is performed $[6,7]$.

While results from translational studies will come out and hopefully will give us better insights in the pathophysiology of bronchiectasis, some steps are urgently needed. A consensus among international experts should be reached on specific definitions of the etiologies of bronchiectasis, including post-infective, COPD-, asthma- or connective tissue disease-related bronchiectasis. We should also promote well-designed, multicenter, prospective studies broadening the spectrum of investigations and including genetic tests for cystic fibrosis and alpha $_{1}$-antytripsin deficien$\mathrm{cy}$, screening for ciliary dysfunction, second-level immunological tests and functional antibodies, serological tests for autoimmune disease, questionnaire to screen for chronic sinusopathy or GORD, and sputum culture for mycobacteria in all the patients enrolled $[8,9]$. From a clinical perspective, it might also justify a large bundle of tests to be present among the SOPs of a tertiary care bronchiectasis clinic, balancing the costs of these tests against the possibility to identify a treatable cause of bronchiectasis.

\section{KARGER}

(C) 2017 S. Karger AG, Basel

E-Mail karger@karger.com

www.karger.com/res
Stefano Aliberti, MD, Department of Pathophysiology and Transplantation University of Milan, Cardio-Thoracic Unit and Cystic Fibrosis Adult Center Fondazione IRCCS Cà Granda Ospedale Maggiore Policlinico

Via Francesco Sforza 35, IT-20122 Milan (Italy)

E-Mail stefano.aliberti@unimi.it 


\section{References}

1 Buscot M, Pottier H, Marquette CH, Leroy S: Phenotyping adults with non-cystic fibrosis bronchiectasis: a 10-year cohort study in a French regional university hospital center. Respiration 2016;92:1-8.

-2 Lonni S, Chalmers JD, Goeminne PC, McDonnell MJ, Dimakou K, De Soyza A, Polverino E, Van de Kerkhove C, Rutherford R, Davison J, Rosales E, Pesci A, Restrepo MI, Torres A, Aliberti S: Etiology of non-cystic fibrosis bronchiectasis in adults and its correlation to disease severity. Ann Am Thorac Soc 2015;12:1764-1770.

-3 Chalmers JD, Aliberti S, Polverino E, Vendrell M, Crichton M, Loebinger M, Dimakou K, Clifton I, van der Eerden M, Rohde G, Murris-Espin M, Masefield S, Gerada E, Shteinberg M, Ringshausen F, Haworth C, Boersma W, Rademacher J, Hill AT, Aksamit T, O’Donnell A, Morgan L, Milenkovic B, Tramma L, Neves J, Menendez R, Paggiaro P, Botnaru V, Skrgat S, Wilson R, Goeminne P, De Soyza A, Welte T, Torres A, Elborn JS, Blasi F: The EMBARC European Bronchiectasis Registry: protocol for an international observational study. ERJ Open Res 2016;2:pii: 000812015.

-4 Gao YH, Guan WJ, Liu SX, Wang L, Cui JJ, Chen RC, Zhang GJ: Aetiology of bronchiectasis in adults: a systematic literature review. Respirology 2016;21:1376-1383.
5 Hurst JR, Elborn JS, De Soyza A; BRONCH-UK Consortium: COPDbronchiectasis overlap syndrome. Eur Respir J 2015;45:310-313.

6 Pasteur MC, Bilton D, Hill AT; British Thoracic Society Bronchiectasis non-CF Guideline Group: British Thoracic Society guideline for non-CF bronchiectasis. Thorax 2010;65(suppl 1):i1-i58.

7 Pulmonology Portuguese Society Bronchiectasis Study Group: Recommendations for aetiological diagnosis of bronchiectasis. Rev Port Pneumol (2006) 2016;22:222-235.

8 Aliberti S, Masefield S, Polverino E, De Soyza A, Loebinger MR, Menendez R, Ringshausen FC, Vendrell M, Powell P, Chalmers JD; EMBARC Study Group: Research priorities in bronchiectasis: a consensus statement from the EMBARC Clinical Research Collaboration. Eur Respir J 2016;48:632-647.

-9 McDonnell MJ, Aliberti S, Goeminne PC, Restrepo MI, Finch S, Pesci A, Dupont LJ, Fardon TC, Wilson R, Loebinger MR, Skrbic D, Obradovic D, De Soyza A, Ward C, Laffey JG, Rutherford RM, Chalmers JD: Comorbidities and the risk of mortality in patients with bronchiectasis: an international multicentre cohort study. Lancet Respir Med 2016;4:969979. 